\title{
Effectiveness of adherence to lipid lowering therapy on LDL- cholesterol in patients with very high cardiovascular risk: A real-world evidence study in primary care
}

\author{
Valeria Guglielmi a, c, 1 , Alfonso Bellia ${ }^{\mathrm{a}, 1}$, Serena Pecchioli ${ }^{\mathrm{b}}$, David Della-Morte ${ }^{\mathrm{a}, \mathrm{c}}$, \\ Damiano Parretti ${ }^{\mathrm{d}}$, Iacopo Cricelli ${ }^{\mathrm{b}}$, Gerardo Medea ${ }^{\mathrm{d}}$, Paolo Sbraccia ${ }^{\mathrm{a}}$, Davide Lauro ${ }^{\mathrm{a}}$, \\ Claudio Cricelli ${ }^{\mathrm{d}}$, Francesco Lapi ${ }^{\text {b, * }}$ \\ a Department of Systems Medicine, University of Rome "Tor Vergata", Italy \\ ${ }^{\mathrm{b}}$ Health Search, Italian College of General Practitioners and Primary Care, Florence, Italy \\ c San Raffaele Roma Open University, Rome, Italy \\ d Italian College of General Practitioners and Primary Care, Florence, Italy
}

\section{A R T I C L E I N F O}

\section{Article history:}

Received 20 March 2017

Received in revised form

18 April 2017

Accepted 17 May 2017

Available online 20 May 2017

\section{Keywords:}

Lipid lowering drugs

High cardiovascular risk

Adherence to statins

Primary care

Low density lipoproteins

Cholesterol

\begin{abstract}
A B S T R A C T
Background and aims: Despite management guidelines advocating statin/ezetimibe use in very high cardiovascular risk (CV) conditions, adherence to this therapy is still suboptimal and LDL-C target attainment unsatisfactory. We aimed to investigate the level of adherence to statin/ezetimibe and LDL-C target achievement rates in an unselected very high CV risk population in primary care setting in Italy. Methods: We performed a retrospective population-based study using the Health Search IMS Health Longitudinal Patient Database (HSD), including adult patients at very high CV risk, newly treated with statin, ezetimibe or their combination, with 3 and 6 months of follow-up.

Results: Although the large majority of patients had previous major CV events (99.9\%), only $61 \%$ and $55.14 \%$ resulted adherent (Proportion of Days Covered, PDC $\geq 80 \%$ ) after 3 and 6 months, respectively. High adherence entailed almost a three times higher probability to reach the therapeutic LDL-C target ( 3 months: $\mathrm{OR}=2.26$ [95\% [CI]: 1.88 to 2.72]; 6-months: $\mathrm{OR}=2.74$ [95\% CI: 2.27 to 3.31]). The odds to treat to LDL-C target was greater for simvastatin-ezetimibe fixed combination, simvastatin, atorvastatin and rosuvastatin, in decreasing order. Finally, poor adherence was slightly more prevalent among patients treated with less effective statins, and at both low and maximal dosage regimens.

Conclusions: This population-based study showed that adherence to statin therapy is poor even among patients who have already experienced a CV event. Failure to achieve recommended LDL-C levels appears imputable to the use of moderate doses and low to standard efficacy statins.
\end{abstract}

(C) 2017 Elsevier B.V. All rights reserved.

\section{Introduction}

Effective lipid-lowering therapy exerts a crucial role for both primary and secondary prevention of atherosclerotic cardiovascular diseases (ACVDs) in patients for whom lifestyle change alone has proven to be insufficient. The main European guidelines on ACVDs prevention in clinical practice recommend to modulate intensity of pharmacological intervention at the individual level,

\footnotetext{
* Corresponding author. Health Search, Italian College of General Practitioners and Primary Care, Via Sestese 61, 50149, Florence, Italy.

E-mail address: lapi.francesco@simg.it (F. Lapi).

1 These authors contributed equally to this work.
}

according to the overall cardiovascular (CV) risk [1].

Thereby, for patients with very high CV risk, namely those with documented ACVD, diabetes mellitus with organ damage, moderate to severe chronic kidney disease, or a calculated 10-year ACVD risk $\geq 10 \%$ according to Systemic COronary Risk Estimation (SCORE) $[2,3]$, the current treatment target advocated for low density lipoprotein cholesterol (LDL-C) is set at $70 \mathrm{mg} / \mathrm{dL}(1.81 \mathrm{mmol} / \mathrm{L})$ or at a $\geq 50 \%$ reduction from relative baseline levels [4,5]. These treatment targets for LDL-C are primarily based on results of a number of randomized clinical trials (RCTs) performed over the last two decades, which reported substantial clinical benefits in terms of risk reduction by this treat-to-target approach in various subgroups of patients at very high CV risk [6]. Among available lipid-lowering 
medications, 3-hydroxy-3-methylglutaryl-coenzyme A (HMG-CoA) reductase inhibitors (statins) have become the cornerstone of hypercholesterolemia treatment in both primary and secondary CV prevention, having shown substantial reductions of LDL-C strongly associated with better CV outcomes. Several major RCTs clearly demonstrated the efficacy of statin therapy with regard to the prevention of CV events in patients after acute coronary syndromes [7] stroke or TIA [8], and in patients with stable coronary artery disease [9], with a weighted average reduction in major vascular events of $24 \%$ per $1 \mathrm{mmol} / \mathrm{L}$ (38.7 $\mathrm{mg} / \mathrm{dl}$ ) reduction in LDL-C, with highly significant reductions in non-fatal myocardial infarction (27\%) and in coronary death (20\%) [6]. On top of statin therapy, additional effect on LDL-C lowering and CV outcomes improvement has been recently demonstrated with ezetimibe, (a selective cholesterol absorption inhibitor), in particular in patients with recent acute coronary syndrome [10].

Despite these well-established major clinical benefits and the abundance of management guidelines advocating statin use in high CV risk conditions [11,12], the direct translation of trial results to clinical practice is still unsatisfactory [13]. In particular, high-dose statin therapy is frequently underused and LDL-C goals are not met in a substantial proportion of high CV risk patients [14-16].

Suboptimal adherence to statins therapy is usually advocated as the main barrier to reach and maintain LDL-C targets [17]. Statins down-titration or discontinuation occurs more frequently among patients who did not tolerate the drug, especially after dose escalation. Several factors may contribute to statin intolerance [18], including patient-related factors, drug-related factors (e.g. dose of administration, polarity of the compound, degradation pathways) and the concomitant use of other drugs, which may account for most of the muscle-related adverse events reported with statins [19]. However, in a significant proportion of patients, musclerelated statin-intolerance cannot be objectively documented by physicians and, therefore, it is frequently set as a psychosomatic disorder [20]. Inappropriate prescribing (e.g. choosing low potency statins when robust lipid lowering effect would be required) can also lead to perceived lack of therapy efficacy and thus suboptimal adherence.

Additionally, irrespective of patient compliance, poor physicians' attention to the different LDL-C reduction rates provided by different statins, and the concomitant presence of some comorbidities limiting the use of high-dose statins in some patients, may also account for failure in reaching LDL-C targets. Given the relevant implications for public health and subsequent economic impact of statin therapy efficacy, the present study aimed to investigate adherence to statin/ezetimibe treatment and LDL-C target achievement rates in a very high CV risk unselected population from primary care setting in Italy, by using the Health Search IMS Health Longitudinal Patient Database (HSD).

\section{Patients and methods}

\subsection{Data source}

We used data from the Italian HSD. This is a general practice database created for research purposes, including patients' demographic details that are linked, by an encrypted code, to clinical records, drug prescriptions, specialist referrals, hospital admissions and date of death. Clinical examinations, drug prescriptions and patients' diseases were coded using the National Health code system, the Anatomical Therapeutic Chemical (ATC) classification system and the International Classification of Diseases 9th Revision Clinical Modification (ICD9CM), respectively. From a network of about one-thousand general practitioners (GPs), who electronically register clinical patient data on a voluntary basis, we identified
700 GPs homogeneously distributed across Italy, covering a population of more than one million individuals, whose data collection fulfilled the "up-to standard" quality criteria. The validity of HSD data for epidemiological research is supported by a number of studies [21-24].

\subsection{Study population}

We selected a cohort of patients aged $\geq 18$ years and treated with statins (ATC code: C10AA*), ezetimibe (ATC codes: C10AX09), ezetimibe-statins fixed combinations (ATC codes: C10BA02, C10BA05, C10BA06) or other fixed combinations including statins (ATC code: C10BA $^{*}$ ), between January 1, 2001 and December 31, 2013. To be eligible, patients had to be classified at very high cardiovascular (CV) risk according to SCORE guidelines (i.e., primary prevention) or with a clinical history of stroke, ischemic cardiomyopathy, peripheral arteriopathy, diabetes with complications, or severe kidney failure (i.e., secondary prevention). Each patient was assessed using demographic and clinical information being registered before or on the index date. Patients in the selected cohort were followed until the occurrence of one of the following events: death from any cause, the 90 days of follow-up, end of data registration with their GP, or end of data availability (December 31, 2013). Those patients with less than 90-day follow-up were, therefore, excluded. In addition, we formed two further cohorts by using the same aforementioned criteria and extending follow-up to 180 and 365 days.

\subsection{Outcomes definition}

We operationally defined patients at target whether their level of LDL-C was lower than $70 \mathrm{mg} / \mathrm{dL}(1.81 \mathrm{mmol} / \mathrm{L})$ during the six months following the end of follow-up. This cut-off value was based on current clinical guidelines [1] and the reimbursement criteria adopted by the Italian Medicines Agency (AIFA) through NOTA 13 [25]. In case of more LDL-C measurements, we adopted the highest recorded value to classify patients. If LDL-C was not registered, all the available measures of HDL cholesterol (HDL-C), total cholesterol (TC), and triglycerides being reported in the same year were used to derive LDL-C (mg/dl) according to the Friedewald formula: $\mathrm{LDL}-\mathrm{C}=[\mathrm{TC}(\mathrm{mg} / \mathrm{dl})-\mathrm{HDL}(\mathrm{mg} / \mathrm{dl})]-1 / 5^{*}$ triglycerides $(\mathrm{mg} / \mathrm{dl})[26]$.

\subsection{Exposure definition}

We identified adherers and non-adherers to statins/ezetimibe using the Proportion of Days Covered (PDC). PDC is calculated by dividing the cumulative days of medication use by the length of follow-up. The duration of each prescription was gathered by dividing the total prescribed mgs of statin/ezetimibe for the related Prescribed Daily Dosage (PDD). Concerning overlapping prescriptions, patients were assumed to have refilled early and not completed the first prescription before starting the following prescription. A patient was, therefore, considered treated continuously if the duration of the interval between two prescriptions was within a time period shorter than 30 days. Otherwise, when medications were not taken continuously or were interrupted, a "grace period" of 30 days was added to the last presumed day of exposure. The grace period is an additional time covered by therapy, the duration of which depends on the pharmacological properties of the drug as well as by the prescription pattern in general practice. The adoption of a grace period also minimizes the potential bias of the unrecorded in-hospital use of medications. Poor adherence was defined as a PDC $<80 \%$ [27]. 


\subsection{Covariates}

Variables pertaining to risk factors for dyslipidemia, as well as those identified as being potential confounders of the level of adherence-dyslipidemia association, were identified. With regard to patients lifestyle, we took into account the following variables: body mass index (BMI), considering the last BMI measurement during 12 months, before or at the entry date; smoking status, using the last available information to classify patients as nonsmoker, smoker or ex-smoker, before or at the entry date.

As far as the patients' history of cardiovascular diseases is concerned, we adjusted for the clinical history ischemic heart disease, ischemic/hemorrhagic stroke, peripheral vascular diseases, heart failure, hypertension, diabetes (with or without complications), chronic kidney impairment (moderate or severe), atrial fibrillation; familial hypercholesterolemia was recognized by means of the clinical criteria provided by the Dutch Lipid Clinic Network (DLCN) score [28]. We also reported the level of total cholesterol, high- and low-density lipoproteins, and levels of triglycerides. Finally, concomitant treatments, prescribed during the year preceding or at the index date, were also taken into account as potential confounders of the level of adherence. These medications include antihypertensive drugs, previous use of statins/ezetimibe, antidiabetic drugs and low-dose aspirin.

\subsection{Data analysis}

Variables were analyzed descriptively. In the primary analysis, univariate and multivariate logistic regressions were used to estimate the association between adherence or not to prescribed lipid lowering medications and achievement of LDL-C target below $70 \mathrm{mg} / \mathrm{dl}$. In addition to age and sex, logistic regression was adjusted for the risk factors and confounders listed above. Odds ratios (ORs) and related 95\% confidence intervals $(\mathrm{CI})$ were given as measures of association. This multivariable analysis was re-run taking into account a follow-up period up to 180 and 365 days. In the secondary analysis, selected individual medications were entered in the models, assuming that those patients prescribed with the same drug for more than half ( $>45$ days) of the follow-up period were categorized as individual users. For this analysis, we excluded patients exposed to lipid lowering drugs during the year before or at the index date. We also estimated proportions of patients not achieving the LDL-C target during the follow-up period with regard to different dosages of prescribed drugs. Finally, sensitivity analysis was performed to evaluate the effect of the inclusion of prevalent users on the results. For this purpose, we rerun the primary analysis having excluded those patients exposed to lipid lowering drugs before the index date.

\section{Results}

Table 1 shows baseline demographics and clinical features of patients who met the eligibility criteria $(n=18423)$. Male gender was predominant (56\%), with a mean age of $66.6 \pm 0.4$ years. The majority of patients were overweight as demonstrated by the mean body mass index (BMI), whereas only $0.54 \%$ of subjects had $\mathrm{BMI}<20$. Proportion of current smokers was also substantial (nearly $8 \%$ ), especially taking into account the very high CV risk of this selected population.

This population was inclusive of patients with familial hypercholesterolemia defined as definite $(12.5 \%)$, probable $(0.46 \%)$ or possible (12.35\%) according to DLCN diagnostic criteria. The large majority of this cohort was composed of patients who had previously experienced a major cardiovascular event (i.e. secondary prevention), defined as previous myocardial infarction (39.1\%),
Table 1

Clinical features of the study population.

\begin{tabular}{|c|c|}
\hline \multirow[t]{2}{*}{ Overall } & \multirow{2}{*}{$\frac{N(\%)^{a}}{18423}$} \\
\hline & \\
\hline Age (years) (mean (SD)) & $66.6(0.4)$ \\
\hline \multicolumn{2}{|l|}{ Sex } \\
\hline Males & $10371(56.29)$ \\
\hline Females & $8052(43.71)$ \\
\hline BMI $\left(\mathrm{Kg} / \mathrm{m}^{2}\right)($ mean $(\mathrm{SD}))$ & $29.23(0.16)$ \\
\hline $\mathrm{BMI}<20$ & $100(0.54)$ \\
\hline Current smoking & $1498(8.13)$ \\
\hline Familial hypercholesterolemia (definite) & $13(0.07)$ \\
\hline Familial hypercholesterolemia (probable) & $85(0.46)$ \\
\hline Familial hypercholesterolemia (possible) & $2276(12.35)$ \\
\hline Diabetes without complications & $1588(8.62)$ \\
\hline Diabetes with complications & $6824(37.04)$ \\
\hline Stroke & $4556(24.73)$ \\
\hline Ischemic cardiopathy & $7219(39.18)$ \\
\hline Periferal arteriopathy & $2563(13.91)$ \\
\hline Aortocoronary bypass & $503(2.73)$ \\
\hline Chronic kidney failure (moderate) & $3024(16.41)$ \\
\hline Chronic kidney failure (severe) & $381(2.07)$ \\
\hline Atrial fibrillation & $697(3.78)$ \\
\hline Hypertension & $6443(34.97)$ \\
\hline Hypercholesterolemia (excluding any familial dyslipidemia) & $6856(37.21)$ \\
\hline Total cholesterol $(\mathrm{mg} / \mathrm{dl})^{\mathrm{b}}($ mean $(\mathrm{SD}))$ & $229.7(1.2)$ \\
\hline LDL cholesterol $(\mathrm{mg} / \mathrm{dl})^{\mathrm{b}}($ mean $(\mathrm{SD}))$ & $149.7(0.8)$ \\
\hline HDL cholesterol (mg/dl) ${ }^{\mathrm{b}}($ mean $(\mathrm{SD}))$ & $51.9(0.3)$ \\
\hline Triglycerides $(\mathrm{mg} / \mathrm{dl})^{\mathrm{b}}($ mean $(\mathrm{SD}))$ & $158.5(0.9)$ \\
\hline Previous use of Lipid lowering drugs & $1566(8.5)$ \\
\hline \multicolumn{2}{|l|}{ Concurrent medications } \\
\hline Antihypertensive drugs & $14715(79.87)$ \\
\hline Low-dose aspirin & $9828(53.35)$ \\
\hline Antidiabetic drugs & $5312(28.83)$ \\
\hline Insulin & $916(4.97)$ \\
\hline
\end{tabular}

previous coronary revascularization (3.46\%), previous coronary artery by-pass graft $(2.73 \%)$ or stroke $(24.7 \%)$. With regard to the other clinical variables assigning a very high CV risk [3], 37\% and $2 \%$ of patients had complicated type 2 diabetes and severe chronic kidney disease, respectively. The whole cohort also included subjects affected by hypertension (34.9\%), uncomplicated type 2 diabetes $(8.6 \%)$, peripheral vascular disease (13.9\%), atrial fibrillation (3.8\%) and moderate renal failure (16.4\%). At study entry, mean total cholesterol, LDL-C and HDL-C levels were $229.7 \pm 1.2 \mathrm{mg} / \mathrm{dl}$, $149.7 \pm 0.8 \mathrm{mg} / \mathrm{dl}$ and $51.9 \pm 0.3 \mathrm{mg} / \mathrm{dl}$, respectively. Of note, only a small proportion of patients had been previously prescribed lipid lowering drugs prior to the index date (8.5\%).

As shown in Table 2, only $61 \%$ and $55.1 \%$ of subjects resulted adherent ( $P D C \geq 80 \%$ ) to the prescribed lipid-lowering treatments after 3 and 6 months, respectively. As expected, treatment adherence entailed a greater probability to be 'at-target' compared with no adherence, as shown by the adjusted odds ratios at $3-(\mathrm{OR}=2.26$ [95\% [CI]: 1.88 to 2.73 ] and 6-months (OR $=2.76$ [95\% CI: 2.28 to 3.33]) of follow-up. Of note, estimated crude and adjusted ORs were very similar, irrespective of the considered follow-up period.

Table 3 shows the odds to treat to LDL-C target by the different lipid-lowering treatments (in case of adherence), which, as indicated by respective crude and adjusted ORs, was greater for simvastatin-ezetimibe fixed combination, simvastatin, atorvastatin and rosuvastatin, in decreasing order. In addition, mixed statin/ ezetimibe therapies (e.g. in patients who switched from the index treatment to another one during the follow-up period for whatever reason) showed a strong association, though not adjusted, with LDL-C goal achievement (crude OR 2.82; 95\% CI: 1.3-6.1). 
Table 2

Relationships between adherence to lipid lowering drugs and attainment of LDL-C targets as per the current guidelines, after three and six months of follow-up.

\begin{tabular}{lll}
\hline & $\mathbf{N}(\%)$ & $\frac{\text { OR (95\% CI) }}{\text { Crude }}$ \\
\hline 3-month follow-up & & Adjusted \\
$\quad$ All subjects with PDC $<80 \%$ & & Ref. \\
All subjects with PDC $\geq 80 \%$ & $11238(61.00)$ & $2.46(2.24-2.69)$ \\
6-month follow-up & & Ref. \\
All subjects with PDC $<80 \%$ & $9905(55.14)$ & $3.07(2.80-3.37)$ \\
All subjects with PDC $\geq 80 \%$ & Ref. \\
\hline
\end{tabular}

a For all clinical features as reported in Table 1.

Table 3

Relationships between adherence to different lipid lowering treatments and attainment of LDL-C targets after three months of follow-up.

\begin{tabular}{|c|c|c|c|}
\hline & \multirow[t]{2}{*}{$\mathbf{N}(\%)$} & \multicolumn{2}{|l|}{ OR (95\% CI) } \\
\hline & & Crude & Adjusted $^{\mathrm{a}}$ \\
\hline All subjects with PDC $<80 \%$ & & Ref. & Ref. \\
\hline Simvastatin (PDC $\geq 80 \%$ ) & $\begin{array}{l}4010 \\
(60.30)\end{array}$ & $\begin{array}{l}2.36 \\
(1.99-2.79)\end{array}$ & $\begin{array}{l}2.82 \\
(1.94-4.10)\end{array}$ \\
\hline Atorvastatin (PDC $\geq 80 \%$ ) & $\begin{array}{l}3759 \\
(67.62)\end{array}$ & $\begin{array}{l}2.26 \\
(1.95-2.63)\end{array}$ & $\begin{array}{l}2.14 \\
(1.56-2.93)\end{array}$ \\
\hline Rosuvastatin (PDC $\geq 80 \%$ ) & $\begin{array}{l}1405 \\
(63.15)\end{array}$ & $\begin{array}{l}2.38 \\
(1.90-2.98)\end{array}$ & $\begin{array}{l}1.83 \\
(1.13-2.96)\end{array}$ \\
\hline Pravastatin (PDC $\geq 80 \%)$ & $\begin{array}{l}559 \\
(49.73)\end{array}$ & $\begin{array}{l}1.50 \\
(0.95-2.36)\end{array}$ & $\begin{array}{l}0.70 \\
(0.27-1.81)\end{array}$ \\
\hline Fluvastatin (PDC $\geq 80 \%$ ) & $\begin{array}{l}255 \\
(55.80)\end{array}$ & $\begin{array}{l}1.95 \\
(0.84-4.56)\end{array}$ & - \\
\hline Lovastatin (PDC $\geq 80 \%$ ) & $\begin{array}{l}228 \\
(63.16)\end{array}$ & $\begin{array}{l}2.06 \\
(0.74-5.73)\end{array}$ & - \\
\hline Simvastatin/Ezetimibe (PDC $\geq 80 \%$ ) & $\begin{array}{l}131 \\
(63.90)\end{array}$ & $\begin{array}{l}2.09 \\
(1.05-4.16)\end{array}$ & $\begin{array}{l}4.15 \\
(0.61-28.21)\end{array}$ \\
\hline Ezetimibe (PDC $\geq 80 \%$ ) & $\begin{array}{l}21 \\
(67.74)\end{array}$ & - & - \\
\hline Mixed (PDC $\geq 80 \%)$ & $\begin{array}{l}105 \\
(45.06)\end{array}$ & $\begin{array}{l}2.82 \\
(1.3-6.13)\end{array}$ & - \\
\hline
\end{tabular}

${ }^{\mathrm{a}}$ For all clinical features as reported in Table 1.

Finally, as shown in Fig. 1, adherent patients were mostly treated with moderate, rather than low or high dose statins, irrespective of the follow-up period. Indeed, the most used dosages were $20 \mathrm{mg}$ for atorvastatin and simvastatin (the latter also in the fixed combination with ezetimibe) and $10 \mathrm{mg}$ for rosuvastatin. Only less potent statins (pravastatin, fluvastatin and lovastatin) were used at their highest dosages (40 $\mathrm{mg}$ for pravastatin and lovastatin and $80 \mathrm{mg}$ for fluvastatin) whereas the maximum daily dosage of rosuvastatin (40 mg) was never prescribed (Fig. 1).

In the sensitivity analysis, when we limited the cohort to incident users, the results were consistent with those gathered in the primary analysis (Supplementary Table 1).

\section{Discussion}

To our knowledge, this is the first study estimating adherence to statin, ezetimibe or their combination treatments and LDL-C target achievement rates, in a representative sample of the Italian general population at very high CV risk. These findings showed that these patients do not frequently receive and maintain appropriate highintensity lipid lowering therapy in primary care.

Although low to moderate intensity statin therapy has been shown to be highly effective in the majority of patients [29], highdose statins provide additional clinical benefits for secondary prevention of $C V$ reoccurrence in those patients who have already experimented an acute event or are at very high clinical risk [30]. Nevertheless, widespread use of suboptimal-intensity lipid lowering therapy in high-risk populations has been already reported, attributable to a number of reasons essentially dealing with reduced adherence of patients to prescribed drugs and therapeutic inertia of physicians [31,32]. Indeed, most patients included in our cohort were on low potency statins, frequently at low to moderate dosages, and combination therapy with statin-ezetimibe was also infrequently prescribed. That being said, non-adherence to prescribed drugs is an ongoing issue in the management of hypercholesterolemia [33], as patients with established CVD who discontinue statins have been demonstrated to be at increased mortality risk compared with those who regularly assume the medications. In our very high-risk population, only $61 \%$ and $55.14 \%$ of subjects were adherent to statin therapy after 3 and 6 months from the first drug prescription, respectively, confirming that adherence to these medications is suboptimal even among patients who have already experienced a CV event [15].

In clinical practice, underuse and discontinuation of medications for chronic diseases are common occurrence in most therapeutic areas. In our cohort, poor adherence was slightly more prevalent among patients treated with less potent (in terms of plasma LDL-C lowering effect) statin therapies, as well as in those at extreme (both minimal and maximal) dosage regimens. Therefore, the underlying reasons for lower adherence rates seem to range from perceived lack of therapeutic benefit (and, thus, inappropriate prescribing) to concerns about adverse effects or their real onset. High adherence to therapy entailed almost a three-time higher probability to reach the therapeutic LDL-C target as defined by the current European guidelines [1]. Nevertheless, approximately one fourth of the highly adherent cohort also failed to achieve adequate 

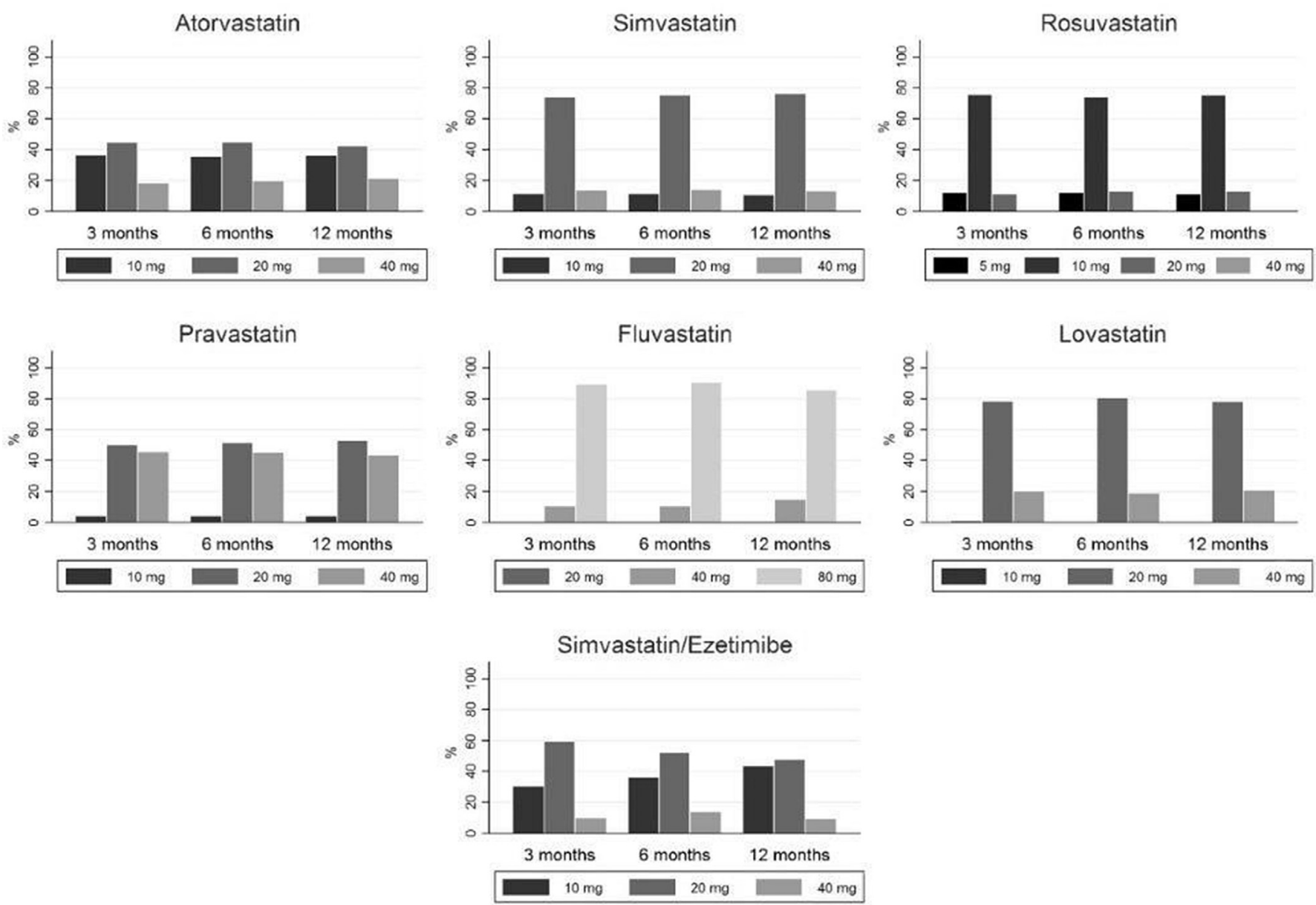

Fig. 1. Proportions of usage of different statins, stratified by dosage and duration of follow-up, among adherent patients (PDC $\geq 80 \%$ ).

LDL-C reduction. In spite of high adherence to therapy, failure to achieve recommended LDL-C levels might be imputable to the use of moderate doses and low to standard efficacy statins [34]. After all, although resorting to high dose statin therapy in such hard clinical outcomes could be detrimental for patient adherence, due to a higher frequency of side effects, on the other hand, it is critical in terms of LDL-C target achievement. The proportions of 'not-attarget' patients almost invariably slightly decreased during followup, highlighting the positive impact of treatment duration on LDL-C advocated reduction.

This study has several strengths. First, HSD is currently one of the largest general practice databases in Europe, which allowed us to adjust for a number of important confounders in a cohort of patients at very high CV risk and lipid-lowering drug users. Second, in the HSD, information on drug exposure is prospectively recorded, thus minimizing any recall bias. Third, the fact that we were able to identify patient's PDDs instead of DDDs (as used in claims databases), sensibly reduced exposure misclassification in light of the great heterogeneity in terms of prescribed doses for this medication class.

This study has limitations as well. First, the fact that HSD collects information about the writing instead of filling of prescriptions may have led to an underestimation of the associations. However, the relationships we found were statistically significant. Furthermore, we included very high-CV-risk patients, assuming that these drugs are actually taken by most of the patients. Second, the inclusion of prevalent users might have biased the results towards the null. Also in this case, we were able to capture statistically significant associations, and as we excluded the $8,5 \%$ of prevalent users from the cohort, our findings were expectedly in line with those obtained for the primary analysis.

In conclusion, our findings indicate a substantial underuse of statin therapy among patients at very high risk for CV events, for whom "aggressive" strategies targeted to reduce this risk are highly recommended. Concomitant overuse of moderate to low intensity statin therapy and general poor adherence to lipid lowering therapy result in a large number of patients who are inadequately protected from recurrent CV events. In this context, Italian health care system-based interventions aimed to improve awareness of this issue in both patients and physicians are highly necessary. Further, development and access to the market of new lipidlowering drugs, such as the new agents targeting proprotein convertase subtilisin/kexin type 9 (PCSK9), with the additional advantage of long-term bioavailability and simplified regimen of administration, could help improve medication adherence, optimize patient care and eventually improve clinical outcomes.

\section{Conflict of interest}

I. Cricelli and F. Lapi provided consultancies in protocol preparation for epidemiological studies and data analyses for Sanofi and Amgen. C. Cricelli, D. Parretti and G. Medea provided clinical consultancies for Sanofi and Amgen. V. Guglielmi, A. Bellia, S. Pecchioli, D. Della-Morte, P. Sbraccia and D. Lauro declare no conflict of 
interests.

\section{Financial support}

This work was supported by Amgen.

\section{Author contributions}

F.L. conceived and designed the research; F.L., S.P. performed statistical analysis; V.G., A,B., F.L., drafted the manuscript; S.P., I.C., G.M., D.P. acquired the data; I.C., C.C. handled funding; I.C., G.M., D. P, C.C., D. D-M., P.S., D.L. made critical revision of the manuscript for key intellectual content; C.C. supervised the research.

\section{Appendix A. Supplementary data}

Supplementary data related to this article can be found at http:// dx.doi.org/10.1016/j.atherosclerosis.2017.05.018.

\section{References}

[1] A.L. Catapano, I. Graham, G. De Backer, et al., 2016 ESC/EAS guidelines for the management of Dyslipidaemias: the task force for the management of Dyslipidaemias of the european society of cardiology (ESC) and european atherosclerosis society (EAS)Developed with the special contribution of the european assocciation for cardiovascular prevention \& rehabilitation (EACPR), Eur. Heart J. 37 (39) (2016 Oct 14) 2999-3058.

[2] R.M. Conroy, K. Pyorala, A.P. Fitzgerald, et al., Estimation of ten-year risk of fatal cardiovascular disease in Europe: the SCORE project, Eur. Heart J. 24 (2003) 987-1003.

[3] M.F. Piepoli, A.W. Hoes, S. Agewall, et al., 2016 european guidelines on cardiovascular disease prevention in clinical practice: the sixth joint task force of the european society of cardiology and other societies on cardiovascular disease prevention in clinical practice (constituted by representatives of 10 societies and by invited experts): Developed with the special contribution of the european association for cardiovascular prevention \& rehabilitation (EACPR), Eur. J. Prev. Cardiol. 23 (11) (2016 Jul). NP1-NP96, http://dx.doi/10. $1177 / 2047487316653709$.

[4] T.A. Jacobson, M.K. Ito, K.C. Maki, et al., National Lipid Association recommendations for patient-centered management of dyslipidemia: part 1executive summary, J. Clin. Lipidol. 8 (2014) 473-488.

[5] T.J. Anderson, J. Gregoire, R.A. Hegele, et al., 2012 update of the Canadian Cardiovascular Society guidelines for the diagnosis and treatment of dyslipidemia for the prevention of cardiovascular disease in the adult, Can. J. Cardiol. 29 (2013) 151-167.

[6] C. Baigent, L. Blackwell, J. Emberson, et al., Efficacy and safety of more intensive lowering of LDL cholesterol: a meta-analysis of data from 170,000 participants in 26 randomised trials, Lancet 376 (2010) 1670-1681.

[7] C.P. Cannon, E. Braunwald, C.H. McCabe, et al., Intensive versus moderate lipid lowering with statins after acute coronary syndromes, N. Engl. J. Med. 350 (2004) 1495-1504.

[8] P. Amarenco, J. Bogousslavsky, A. Callahan 3rd, et al., High-dose atorvastatin after stroke or transient ischemic attack, N. Engl. J. Med. 355 (2006) 549-559.

[9] J.C. LaRosa, S.M. Grundy, D.D. Waters, et al., Intensive lipid lowering with atorvastatin in patients with stable coronary disease, N. Engl. J. Med. 352 (2005) 1425-1435.

[10] C.P. Cannon, M.A. Blazing, R.P. Giugliano, et al., Ezetimibe added to statin therapy after acute coronary syndromes, N. Engl. J. Med. 372 (2015) 2387-2397.

[11] Executive Summary of The Third Report of The National Cholesterol Education Program (NCEP) Expert Panel on Detection, Evaluation, and treatment of high blood cholesterol in adults (adult treatment panel III), JAMA 285 (2001) 2486-2497.
[12] G. De Backer, E. Ambrosioni, K. Borch-Johnsen, et al., European guidelines on cardiovascular disease prevention in clinical practice. Third joint task force of european and other societies on cardiovascular disease prevention in clinical practice, Eur. Heart J. 24 (2003) 1601-1610.

[13] S.E. Hoeks, W.J. Scholte op Reimer, Y.R. van Gestel, et al., Medication underuse during long-term follow-up in patients with peripheral arterial disease, Circ. Cardiovasc Qual. Outcomes 2 (2009) 338-343.

[14] T.A. Pearson, I. Laurora, H. Chu, et al., The lipid treatment assessment project (L-TAP): a multicenter survey to evaluate the percentages of dyslipidemic patients receiving lipid-lowering therapy and achieving low-density lipoprotein cholesterol goals, Arch. Intern Med. 160 (2000) 459-467.

[15] Lifestyle and risk factor management and use of drug therapies in coronary patients from 15 countries, Principal results from EUROASPIRE II euro heart survey programme, Eur. Heart J. 22 (2001) 554-572.

[16] Z. Reiner, G. De Backer, Z. Fras, et al., Lipid lowering drug therapy in patients with coronary heart disease from 24 European countries - findings from the EUROASPIRE IV survey, Atherosclerosis 246 (2016) 243-250.

[17] J.S. Benner, R.J. Glynn, H. Mogun, et al., Long-term persistence in use of statin therapy in elderly patients, JAMA 288 (2002) 455-461.

[18] H.E. Bays, P.H. Jones, C.E. Orringer, et al., National lipid association annual summary of clinical lipidology 2016, J. Clin. Lipidol. 10 (2016) S1-S43.

[19] A. Pirillo, A.L. Catapano, Statin intolerance: diagnosis and remedies, Curr. Cardiol. Rep. 17 (2015) 27.

[20] S.E. Nissen, E. Stroes, R.E. Dent-Acosta, et al., Efficacy and tolerability of evolocumab vs ezetimibe in patients with muscle-related statin intolerance: the GAUSS-3 randomized clinical trial, JAMA 315 (2016) 1580-1590.

[21] C. Cricelli, G. Mazzaglia, F. Samani, et al., Prevalence estimates for chronic diseases in Italy: exploring the differences between self-report and primary care databases, J. Public Health Med. 25 (2003) 254-257.

[22] G. Trifiro, P. Morabito, L. Cavagna, et al., Epidemiology of gout and hyperuricaemia in Italy during the years 2005-2009: a nationwide populationbased study, Ann. Rheum. Dis. 72 (2013) 694-700.

[23] G. Giussani, C. Cricelli, F. Mazzoleni, et al., Prevalence and incidence of epilepsy in Italy based on a nationwide database, Neuroepidemiology 43 (2014) $228-232$.

[24] F. Lapi, M. Simonetti, R. Michieli, et al., Assessing 5-year incidence rates and determinants of osteoporotic fractures in primary care, Bone 50 (2012) 85-90.

[25] http://www.agenziafarmaco.gov.it/sites/default/files/Nota13_GU156_ 08072014.pdf, In.

[26] R.S. Niedbala, K.J. Schray, R. Foery, et al., Estimation of low-density lipoprotein by the Friedewald formula and by electrophoresis compared, Clin. Chem. 31 (1985) 1762-1763.

[27] S. Karve, M.A. Cleves, M. Helm, et al., Good and poor adherence: optimal cutpoint for adherence measures using administrative claims data, Curr. Med. Res. Opin. 25 (2009) 2303-2310.

[28] V. Guglielmi, A. Bellia, S. Pecchioli, et al., What is the actual epidemiology of familial hypercholesterolemia in Italy? Evidence from a National Primary Care Database, Int. J. Cardiol. 223 (2016) 701-705.

[29] C. Baigent, A. Keech, P.M. Kearney, et al., Efficacy and safety of cholesterollowering treatment: prospective meta-analysis of data from 90,056 participants in 14 randomised trials of statins, Lancet 366 (2005) 1267-1278.

[30] C.P. Cannon, B.A. Steinberg, S.A. Murphy, et al., Meta-analysis of cardiovascular outcomes trials comparing intensive versus moderate statin therapy, J. Am. Coll. Cardiol. 48 (2006) 438-445.

[31] P.P. Toth, J.M. Foody, J.E. Tomassini, et al., Therapeutic practice patterns related to statin potency and ezetimibe/simvastatin combination therapies in lowering LDL-C in patients with high-risk cardiovascular disease, J. Clin. Lipidol. 8 (2014) 107-116.

[32] R.J. Simpson Jr., K. Tunceli, D.R. Ramey, et al., Treatment pattern changes in high-risk patients newly initiated on statin monotherapy in a managed care setting, J. Clin. Lipidol. 7 (2013) 399-407.

[33] K. Herttua, P. Martikainen, G.D. Batty, et al., Poor adherence to statin and antihypertensive therapies as risk factors for fatal stroke, J. Am. Coll. Cardiol. 67 (2016) 1507-1515.

[34] C.E. Chiang, J. Ferrieres, N.N. Gotcheva, et al., Suboptimal control of lipid levels: results from 29 countries participating in the centralized Pan-Regional surveys on the undertreatment of Hypercholesterolaemia (CEPHEUS), J. Atheroscler. Thromb. 23 (2016) 567-587. 